\title{
CT computerised stereotactic biopsy for low density CT lesions presenting with epilepsy
}

\author{
J N WILDEN, P J KELLY \\ From the Department of Neurosurgery, Wessex Neurological Centre, Southampton, UK, and the Department of \\ Neurosurgery, Mayo Clinic, Rochester, USA
}

SUMMARY Thirty five patients presenting with epilepsy alone and non-enhancing low-density lesion on the CT scan underwent a computer-assisted CT-guided stereotactic biopsy with stereotactic angiographic control. There was no mortality or morbidity in this series and the diagnostic yield was $97 \%$. Thirty four patients had low grade intra-axial neoplasms. After an estimation of the pathological extent of the tumour, three patients underwent a computer-assisted stereotactic laser resection and 28 patients had radiotherapy.

Patients presenting with epilepsy alone and a low density lesion on CT scan pose three problems: firstly, whether it is safe to perform a biopsy in the absence of neurological signs; secondly, whether the diagnostic yield justifies the risk of biopsy; thirdly, whether the histological diagnosis is likely to alter management, that is, result in a potentially curable resection or radiotherapy. The purpose of this paper is to show that CT computerised stereotactic biopsy with angiographic control is a safe procedure for obtaining a histological diagnosis in this group of patients. Furthermore, with the aid of CT and magnetic resonance imaging (MRI), multiple serial biopsies, together with histological examination of smear preparations and haematoxylin and eosin sections, establish a better estimation of the true pathological limits of the tumour than can be obtained by craniotomy. This information allows a more accurate decision to be made regarding whether curative surgical resection is feasible, or whether radiotherapy should be given.

\section{Methods}

\section{(a) Patients}

Thirty five patients presented to one of us (PJK) at the Mayo Clinic, Rochester, USA, between June 1984 and May 1986. Referrals were from other neurosurgeons and neurologists. At the time of presentation all patients suffered from epilepsy but no other neurological symptoms. Neurological

Address for reprint requests: Mr J Wilden, Wessex Neurological Centre, Southampton General Hospital, Shirley, Southampton SO9 4XY, UK.

Received 28 October 1986. Accepted 11 February 1987 examination was entirely normal. CT scan showed a low density lesion with no evidence of contrast enhancement.

\section{(b) Data acquisition}

All patients underwent a high resolution contrast CT scar with their heads placed in a modified CT compatible Todd Wells stereotactic frame, ${ }^{1}$ using a transverse localisation sys tem consisting of nine carbon rods in a letter " $N$ " configuration secured anteriorly and laterally to the head. In addition, all patients received an arterial digital subtraction angiography (DSA) with an angiographic reference system attached to the stereotactic head holder. ${ }^{2}$ Twenty-sixo patients underwent a stereotactic MRI, using a compatible stereotactic frame and a similar localisation system with nine rods filled with copper sulphate.

Each CT and MRI slice with the nine localising artefacts and the stereotactic angiogram with localising fiducials was archived onto magnetic tape. The information from the tape was then read into a Data General Eclipse S140 computer system and displayed on an Independent Physicians Diagnostic Console (IPDC), ${ }^{4}$ and in theatre displayed on a Ramtek Raster Terminal.

\section{(c) Surgical planning}

The nine localisation artefacts on each stereotactic CT slice were read and recorded by the computer, with reference to the centre of the localisation system (Xo, Yo, Zo). A target for the biopsy was chosen on the CT slice, and MRI if available, and similarly read and recorded by the computer with reference to the localising artefacts. The target was then represented in three dimensional space and the computer calculated the $\mathrm{X}, \mathrm{Y}$ and $\mathrm{Z}$ adjustments on the stereotactic frame to place the target into the centre of the stereotactic arc-quadrant. In addition, the target was displayed on the stereotactic DSA and a surgical trajectory determined which avoided vascular structures. An axial trajectory on CT and MR image was chosen (fig) in order to simplify histological and imaging correlations. The computer converted the tra- 

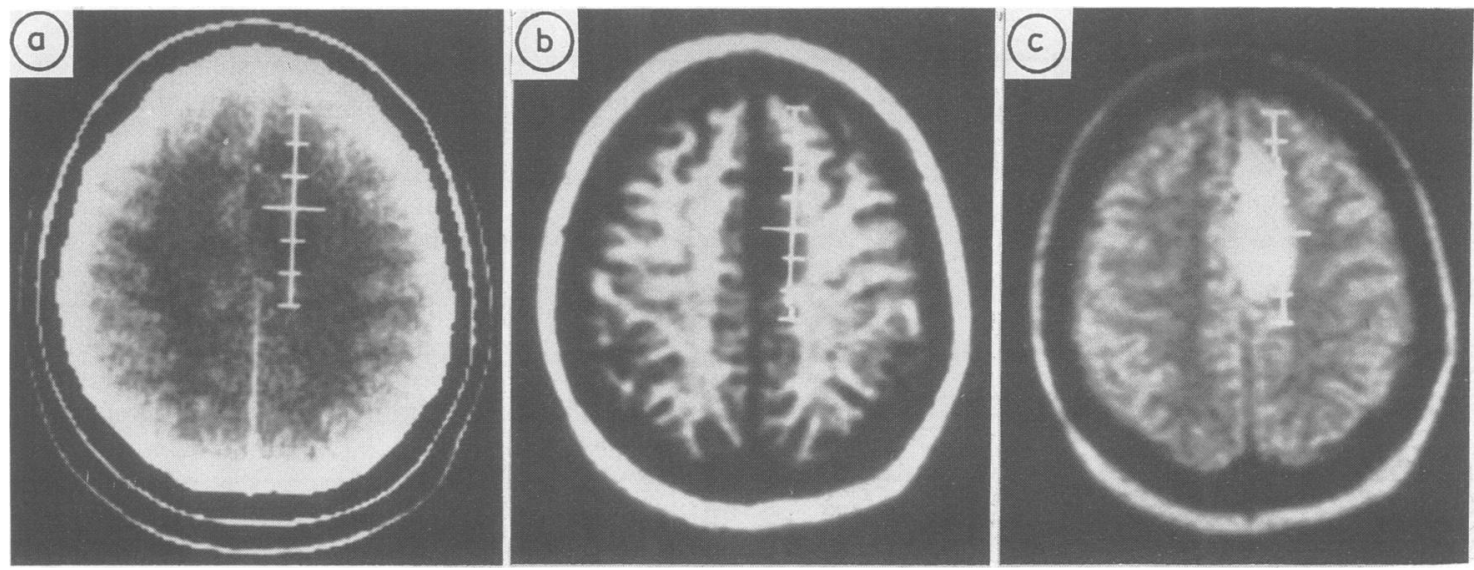

Fig (a) CT scan showing surgical trajectory and multiple biopsy sites through low density lesion. (b) MRI scan (TI weighting) showing similar trajectory. (c) MRI scan (T2 weighting) showing similar trajectory.

jectory into mechanical settings on the arc and collar of the stereotactic frame. Usually, surgical planning was performed the day after data acquisition.

\section{(d) Biopsy procedure}

Under a general endotracheal anaesthetic, the CT compatible stereotactic head frame was reapplied using the same cranial pin sites and micrometer settings that had been used during the data acquisition phase. The stereotactic frame settings $(X, Y, Z)$ determined during surgical planning were set on the frame, and the planned trajectory on the arc and collar. A guide tube was inserted through the collar of the stereotactic frame and AP and lateral radiographs taken to confirm the position of the entry point and compare with the stereotactic angiogram. The skull was opened by a $1 / 8^{\prime \prime}$ $(2.5 \mathrm{~mm})$ diameter air powered drill and the dura penetrated by a unipolar insulated metal tipped diathermy. A 7/64" ( $2 \mathrm{~mm}$ ) wide biopsy cannula with a $10 \mathrm{~mm}$ wide-window was advanced to the nearest margin of the tumour and usually a series of five or six biopsies obtained along the desired trajectory at intervals of $10 \mathrm{~mm}$ until the furthest margin of the tumour was reached as documented on the CT or MRI scan. Each position for the biopsy was confirmed by AP and lateral stereotactic 4 metre teleradiographs.

\section{(e) Pathology}

Immediate haematoxylin and eosin stained smears were prepared from a small part of each biopsy. The rest of the biopsy was fixed in formalin and gluteraldehyde, then paraffin sections prepared with haematoxylin and eosin stains. Each specimen was examined according to the following classification ${ }^{5}$ for: (1) solid tumour tissue characterised by coalescent tumour cells, vascular elements, areas of

Table 1 Main site of low density lesion on CT scan

\begin{tabular}{lllll}
\hline & Hemisphere & Frontal & Temporal & Parietal \\
\hline Right & $10(29 \%)$ & $7(20 \%)$ & $1(3 \%)$ & $2(6 \%)$ \\
Left & $25(71 \%)$ & $16(45 \%)$ & $9(26 \%)$ & $0(0 \%)$ \\
\hline
\end{tabular}

necrosis, with no intact intervening neural parenchyma; (2) infiltrating tumour cells demonstrating large abnormal nuclei and scanty cytoplasm found in intact neural parenchyma; (3) areas of necrosis and (4) normal brain tissue with or without oedema. In addition, degree of anaplasia was classified into four grades. ${ }^{6}$

\section{(f) Extirpation of lesions by computer-assisted stereotactic} laser

Selected patients considered to have circumscribed lesions as detected by CT, MRI scans and biopsy material underwent a stereotactic laser resection as previously described, ${ }^{4}$ provided that it was not likely to result in a neurological deficit, for example, a weakness or language defect.

\section{(g) Radiotherapy}

Some patients received a focal dose of radiation varying between 55 and $64 \mathrm{~Gy}$ to the tumour, and others underwent whole brain irradiation, dosages ranging from 45 to $55 \mathrm{~Gy}$ to the whole brain with a booster dose to the lesion.

\section{Results}

\section{(1) Patients}

Thirty five patients were selected from a total of 258 patients undergoing a CT stereotactic computer assisted biopsy with stereotactic angiographic control. There were 14 females and 21 males with an average age of 41.8 years, ranging from 25 to 73 years. The average duration of epilepsy was 36.9 months ranging from 1 to 156 months. The main sites of the lesion depicted on CT scans are shown in table 1.

\section{(2) Biopsy}

The average number of biopsied sites per case was $4 \cdot 5$. There was no morbidity or mortality in this series and most patients were discharged from hospital within 48 hours of surgery. 
Table 2 Description of pathology

\begin{tabular}{lrrr}
\hline & & No & Per cent \\
\hline Astrocytoma & Grade 1 & 2 & 6 \\
& 2 & 13 & 36 \\
& 3 & 2 & 6 \\
Oligodendroglioma & 4 & 1 & 3 \\
Mixed Oligo-astrocytoma & Grade 2 & 6 & 17 \\
& 3 & 1 & 3 \\
Ganglio-glioma & 2 & 5 & 14 \\
Non-Diagnosed & 3 & 2 & 6 \\
& & 2 & 6 \\
\hline
\end{tabular}

Table 3 Biopsy findings

\begin{tabular}{lrl}
\hline Description of biopsy & No & Per cent \\
\hline Solid tumour only & 6 & 17 \\
Infiltrating cells only & 20 & 57 \\
Solid tumour and infiltrating cells & 8 & 23 \\
Gliosis only & 1 & 3 \\
\hline
\end{tabular}

\section{(3) Pathology}

A pathological diagnosis was obtained in 34 of 35 cases, a yield of $97 \%$ (table 2). Biopsy material from the undiagnosed case revealed only gliosis, two biopsies were obtained from this lesion located in the left temporal lobe. In addition to the biopsies containing solid tumour and infiltrating cells (table 3 ), one biopsy demonstrated necrosis, two oedematous brain and nine showed normal brain.

\section{(4) Radiotherapy and extirpation of lesions by computer-assisted stereotactic laser}

Twenty-eight patients underwent radiotherapy. Sixteen patients received focal dose irradiation to the tumour and 12 underwent whole brain radiotherapy. Three patients underwent extirpation of the lesion using the computer-assisted stereotactic laser. This procedure followed stereotactic biopsy when it was considered that the lesion consisted primarily of solid tumour tissue with little infiltration into parenchyma. One lesion was in the left frontal, another in the left temporal, a third in the right frontal region. In all three patients the biopsy result was confirmed and none developed a neurological deficit.

\section{Discussion}

These results show that patients presenting with epilepsy alone and a low density lesion on CT scan, can safely undergo a CT guided computer-assisted stereotactic biopsy with angiographic control, and that a high diagnostic yield, $97 \%$ in this series, can be obtained. The importance of knowing the pathology, together with the probable limits of the lesion, is that a small percentage of tumours can be resected without neurological deficit, $10 \%$ in this series. Furthermore, pathology is required before consideration of radiotherapy and some patients may wish to be certain about the diagnosis, in order to make suitable adjustments to their life-style.

In the present series of 35 cases, there was no mor- $-\vec{\theta}$ tality or morbidity from patients undergoing a stereo- $D$ tactic biopsy. The authors are unaware of any other published series of stereotactic biopsies in patients $\frac{\mathrm{O}}{\mathrm{O}}$ presenting in a similar fashion. However, in the larger series of CT guided stereotactic biopsies, ${ }^{7}$ usually associated with other procedures including interstitial $\stackrel{\vec{\rho}}{\vec{\rho}}$ irradiation, ${ }^{89}$ and aspiration of cysts, ${ }^{910}$ the mor- $\overline{0}$ tality varied from zero ${ }^{9}$ to $2 \cdot 3 \%^{8}$ and morbidity from 등 $1^{7}$ to $5.9 \% .{ }^{10}$ Deaths in these series were mainly $\frac{\bar{c}}{\bar{c}}$ attributable to biopsy of midline structures ${ }^{7}$ or $\mathbb{D}$ enhancing cerebral lesions resulting in haemorrhage or cerebral swelling, ${ }^{8}$ rather than biopsy of low density hemisphere lesions as described in this series.

Multiple biopsies were obtained under CT guided, computer-assisted stereaxis with stereotactic local- $\stackrel{\omega}{\sigma}$ ising angiography where the computer visually dis- $\overline{\bar{J}}$ played on the angiogram the chosen biopsy target $\overline{0}$ recorded from the CT scan. Whilst there was no mor- or bidity or mortality in this small series, even in a larger $\overrightarrow{\vec{O}}$ series of 261 biopsies obtained by the same teche nique, ${ }^{7}$ the morbidity was less than $1 \%$. The mo $\overrightarrow{\mathrm{C}^{2}}$ bidity compares favourably with other larger series $N$ where the rate is between $3^{8}$ and $5 \cdot 9,{ }^{10}$ when multip鬼 biopsies were taken, but the site of the biopsy target was only visually estimated on angiography rather $O$ than stereotactically computerised and displayed of 0 응 the angiogram. In addition, multiple free hand biop. Ф sies performed without stereotactic guidance usual result in higher levels of morbidity. ${ }^{11}$

The high diagnostic yield of $97 \%$ in this series is the result of two approaches. Firstly, taking multiple biopsies and secondly, the preparation and categorisation of pathological material. The importance of obtaining multiple biopsies is illustrated by the fact that only two biopsies, compared with an average of $\propto$ 4.5 , were obtained in the case that remained $\vec{F}$ undiagnosed.

Pathological categorisation revealed that only $17 \%$ of our cases showed solid tumour alone, consequently examination of peripheral areas for infiltrating tumour or isolated tumour cells is mandatory. In another series ${ }^{12}$ adopting the same pathological categorisation, $75 \%$ of specimens obtained from 3 stereotactic biopsy were diagnosed by the appearance of infiltrating areas or isolated tumour cells.

Comparison of stereotactic biopsy material with subsequent surgical resection on autopsy findings when available, results in a concordance rate of $\frac{D}{O}$ between 84 and $95 \% .^{13-15}$ In this series, of three subsequent extirpations by computer-assisted stereo- $N$ tactic laser, the biopsy results were confirmed by $N$ 
further pathological sampling of the tumour in each case.

The low resection rate in this series possibly reflects a selection from other neurosurgeons and neurologists, who refer patients for biopsy when they consider safe resection is not feasible. Recent review of 461 patients with low grade astrocytoma from the Mayo Clinic ${ }^{16}$ suggested that "total" resection was better than "subtotal" resection and resulted in a "cure" at a 15 year survival rate in the younger age (0-19 year) group. Indeed, stereotactic laser resection of deep-seated low-grade tumours in the younger, less than 20 year age group, also produced a favourable outcome. ${ }^{4}$ Consequently, complete surgical resection of these low-grade astrocytomas is the most satisfactory treatment if it is possible. Hence, early referral is desirable when surgical resection is more feasible and likely to offer a cure.

Twenty-eight patients of this series received greater than $50 \mathrm{~Gy}$ to the lesion. The value of irradiation compared with no treatment has been shown to be beneficial in relatively small series, ${ }^{17}{ }^{18}$ and radiation of greater than $40 \mathrm{~Gy}$ appeared favourable at five year follow-up in the recently reported Mayo Clinic Series. ${ }^{16}$ However, uncertainty remains, consequently the MRC in England and the EORTC in Brussels are jointly organising a multicentre controlled trial of irradiation of low-grade astrocytomas for which a stereotactic or open biopsy will be required.

Finally, given that the overall five year survival rate of low-grade astrocytomas is $40 \%,{ }^{16}$ and the average age of histological diagnosis in this series was 42 years, it is reasonable to suggest that most patients will wish to make suitable changes of life-style and if required, arrangements for dependants. Under these circumstances, a policy of no biopsy but "wait and see", is no longer justified.

JNW acknowledges the support from the Royal College of Surgeons (England) for The New York Travelling Fellowship.

\section{References}

1 Goerss S, Kelly PJ, Kall BA, Alker GJ. A computer tomographic stereotactic adaptation system. Neurosurgery 1982;10:375-9.

2 Kelly PJ, Alker GJ, Kall BA, Goerss S. Method of computed tomography-based stereotactic biopsy with arteriographic control. Neurosurgery 1984;14:172-7.
3 Kelly PJ. Principles of stereotactic surgery: instrumentation and procedural aspects. In: Youmans JR, ed. Neurological Surgery. (in press).

4 Kelly PJ. Computer-assisted stereotaxic laser reduction of intra-axial brain neoplasms. $J$ Neurosurg 1986;64: 427-39.

5 Kelly PJ, Daumas-Duport C, Kispert DB, Kall BA, Schaithauer BW, Illig JJ. Histological and volumetric analysis of untreated intracranial glioma-neoplasms studied by computed tomographic and magnetic resonance imaging-based stereotactic serial biopsies. $J$ Neurosurg (in press).

6 Kernahan JW, Sayre GP. Tumours of the central nervous system. Atlas of Tumour Pathology. Section 10, facsicle 35. 1952. Washington: AFIP.

7 Kelly PJ. Computer-assisted stereotaxis: new approaches for the management of intracranial intra-axial tumours. Neurology 1986;36:535-41.

8 Ostertag CB, Mennel HD, Kiessling M. Stereotactic biopsy of brain tumors. Surg Neurol 1980;14:275-83.

9 Apuzzo MLJ, Sabshin JK. Computed tomographic guidance stereotaxis in the management of intracranial mass lesions. Neurosurgery 1983;12:277-84.

10 Lunsford LD, Martinez AJ. Stereotactic exploration of the brain in the era of computed tomography. Surg Neurol 1984;22:222-30.

11 Shetter AG, Bertucinni TV, Pittman HW. Closed needle biopsy in the diagnosis of intracranial mass lesions. Surg Neurol 1977;8:341-5.

12 Daumas-Duport C, Monsaingeon V, N Guyen JP, Missir O, Szikla G. Some correlations between histological and CT aspects of cerebral gliomas contributing to the choice of significant trajectories for stereotactic biopsies. Acta Neurochir Suppl 1984;33: 185-94.

13 Kleihues P, Volk B, Anagnostopoulos J, Kiessling M. Morphological evaluation of stereotactic brain tumour biopsies. Acta Neurochir Suppl 1984;33: 171-81.

14 Monsaingeon V, Daumas-Duport C, Mann M, Miyahara S, Szikla G. Stereotactic sampling biopsies in a series of 268 consecutive cases validity and technical aspects. Acta Neurochir Suppl 1984;33:195-200.

15 Scerrati M, Rossi GF. The reliability of stereotactic biopsy. Acta Neurochir Suppl 1984;33:201-5.

16 Laws ER, Taylor WF, Clifton MB, Okazaki H. Neurosurgical management of low-grade astrocytoma of the cerebral hemispheres. J Neurosurg 1984;61:665-73.

17 Leibel SA, Sheline GE, Wara WM, Boldrey EB, Nielsen SL. The role of radiation therapy in the treatment of astrocytomas. Cancer 1975;35:1551-7.

18 Fazekas JT. Treatment of grade I and II brain astrocytomas. The role of radiotherapy. Int J Radiat Oncol Biol Phys 1977;2:661-6. 